\title{
Associations between dietary patterns and biomarkers of nutrient status and cardiovascular risk factors among adolescents in Germany: results of the German Health Interview and Examination Survey for Children and Adolescents in Germany (KiGGS)
}

Almut Richter ${ }^{1 *}$ (D), Martina Rabenberg ${ }^{1}$, Julia Truthmann, Christin Heidemann¹, Jutta Roosen², Silke Thiele ${ }^{3}$ and Gert B. M. Mensink ${ }^{1}$

\begin{abstract}
Background: The aim of this study is to analyse prevailing dietary patterns among German adolescents and their associations with biomarkers of nutrient status and cardiovascular risk factors.

Methods: Analyses were based on data from the nationwide, representative Health Interview and Examination Survey for Children and Adolescents in Germany, conducted between 2003 and 2006 (KiGGS baseline). Dietary habits of 12 to 17 year olds (2646 boys and 2551 girls) were determined using 34 food groups assessed with a food frequency questionnaire. Principal component analysis was applied to determine the major dietary patterns. The associations between dietary patterns and biomarkers were analysed using linear regression analyses.

Results: We identified three major dietary patterns among boys and two among girls. Higher scores of the 'healthy' patterns (fruits, salad vegetables, wholemeal bread) were associated with higher levels of serum folate and lower levels of homocysteine among both sexes and higher levels of serum vitamin $B_{12}$ among girls. Conversely, higher scores of the 'western' pattern among boys (salty snacks, burger, French fries) were associated with a lower ferritin level and lower diastolic blood pressure. The 'traditional' pattern among boys (white bread, processed meat, meat) was associated with a lower folate level and the 'western and traditional' pattern among girls (salty snacks, burger, French fries) with lower folate and higher homocysteine levels. No associations between dietary patterns and blood lipids, HbA1c and uric acid were found. The mean age of boys with higher scores in the 'western' pattern was higher, whereas the mean age of girls with higher scores in the 'western and traditional' dietary patterns was lower.
\end{abstract}

Conclusions: Adolescents with higher scores in the 'healthy' dietary patterns had a better nutrient profile. Therefore, healthy dietary patterns should be promoted early in life, with a special focus on the sex differences.

Keywords: Dietary patterns, Adolescents, FFQ, Biomarker, CVD, Nutrient status

\footnotetext{
* Correspondence: RichterA@rki.de

${ }^{1}$ Department of Epidemiology and Health Monitoring, Robert Koch Institute

Berlin, Post box 6502 61D-13302 Berlin, Germany

Full list of author information is available at the end of the article
} 


\section{Background}

Adolescence is a life phase where nutrient intake is particularly important and may also change because of hormonal, cognitive, and emotional changes and an accelerating growth rate [1]. Since adolescents are becoming less dependent on the food choices and purchases of their parents, dietary patterns may change in this stage of life. There are several studies indicating that adolescents often do not meet the recommendations for a healthy diet in Western countries [2-8], including in Germany $[9,10]$. Nutrition early in life has an impact on long term health, especially concerning cardiovascular diseases [11-15]. This is probably related to the fact that food and taste preferences develop during childhood and adolescence and often persist into adulthood [16-21].

A better insight in eating habits is necessary to focus public health policies and nutritional intervention in this life stage.

Analysis of dietary patterns can be used to describe the eating behavior in a population. This can be accomplished by investigating a priori-defined healthy eating indices, which are based on a judgement of appropriateness of the food intake. Previously, we analyzed the association between such dietary indices and biomarkers in the same population [22]. For the current study, we applied principal component analysis (PCA), which is a data driven method and results in patterns that more objectively represent prevailing eating habits of the population.

Many previous studies among adults have shown that dietary patterns are related to biomarkers of cardiovascular risk [23-26]. In contrast to this, analyses of dietary patterns and biomarkers, including biomarkers of nutrient status, among adolescents are scarce. Most are based on cohort studies [27-29], with only two being based on representative health surveys, conducted in Australia [30] and Tunisia [31]. Thus, little is known about dietary patterns and measured biomarkers in adolescence.

Therefore, the aim of this study is to determine dietary patterns among a representative sample of German adolescents using PCA and to examine the associations between dietary patterns and biomarkers of nutrient status and cardiovascular risk factors.

\section{Methods}

\section{Study design and study population}

The target population of the German Health Interview and Examination Survey for Children and Adolescents (KiGGS baseline study) consists of all children and adolescents aged 0 to 17 years, with the exception of those in hospitals, state institutions, or foster homes. The survey was conducted by the Robert Koch Institute. The design, sampling strategy, and study protocol have been described elsewhere in detail [32]. Briefly, the sample was drawn using a two-stage clustered and stratified sampling procedure. In the first stage, 167 sample points representative of German communities were selected with regard to community size and federal state. In the second stage, for every age, participants were randomly selected from the local population registries. The survey was approved by the Federal Office for Data Protection and the Charité-Universitätsmedizin Berlin ethics committee. Participants aged 14 years or older and all parents provided written informed consent before the interview and examination procedures. The overall response rate was $66.6 \%$ [32].

\section{Data collection}

The 167 sample points were covered by four study teams between May 2003 and May 2006. Parents and participants older than 11 years of age were asked to complete different self-administered questionnaires in the study centres [33]. These included questions on socio-demographic characteristics as well as health and health related behaviours. In addition, participants underwent a computer-assisted medical interview and a physical examination (e.g. body weight and height measurement) conducted by trained staff. Lastly, nonfasting blood and urine samples were also obtained [34].

\section{Dietary assessment}

Participants aged 12-17 years were further asked to complete a self-administered, semi-quantitative food frequency questionnaire (FFQ) as well. To cover the most relevant food groups for this population group, the semi-quantitative FFQ was developed by the Robert Koch Institute in consultation with several experts in the field of dietary assessment among children and adolescents. The development of the FFQ is described in detail elsewhere [35]. The FFQ was validated against the modified dietary history method DISHES (Dietary Interview Software for Health Examination Studies) and showed fair to moderate ranking validity for food intake amounts for most of the food items (Spearman correlation coefficients from .35 to .69 with most values above .50) [22]. The FFQ included questions on the average food consumption frequency, as well as the average consumed portion size, for 45 food items in the last few weeks. Categories for frequencies were identical for all food items: never; once per month; 2-3 times per month; $1-2$ times per week; $3-4$ times per week; 5-6 times per week; once per day; 2-3 times per day; 4-5 times per day; more than 5 times per day. Foodspecific portion sizes were assessed by five categories and often illustrated with pictures, e.g. using standard household measures (cups, spoons, etc.). Food frequency information was recoded into frequency consumption of these foods per month ( 1 month was set equal to 4 weeks; for example, once per week $=4$, once per day $=28$, more than five times per day $=168$ ). For frequency bands such as one or two times per day, the arithmetic mean was used. 
Portion sizes were converted into equivalent gram amounts using the standard portion sizes provided in the FFQ. The average food intake was then calculated by multiplying the recoded frequencies and portion sizes (average food intake $=$ food frequency (per month) $\mathrm{x}$ portion size $(\mathrm{g})$ ).

If the frequency of consumption was given, but information on portion size was missing, the middle category of portion size provided in the FFQ was imputed as it represents the most frequently chosen portion size for this age group. If the food frequency was missing, then the food item was considered as not having been consumed (average food intake $=$ zero).

Food items were grouped into 34 food groups, according to a former dietary pattern analysis based on a modified diet history interview (DISHES) in a subgroup of this study population [36].

Total energy intake was calculated by multiplying the intake and mean energy contents of the FFQ food items. The energy content of every food group was calculated based on weighted estimates of consumption frequencies of specific foods within the food groups based on the comprehensive DISHES data (e.g. several different breads on the total amount of bread).

\section{Assessment of biomarkers}

Several biomarkers were measured using the blood and urine samples collected in KiGGS. Venous blood samples were obtained from the participants if the parents and the adolescents themselves gave consent. Serum was separated and transported on dry ice to a central laboratory according to a standardised protocol. Samples were kept at $-40{ }^{\circ} \mathrm{C}$ until analysed. Pre-analytic and analytic standards have been previously described in detail [34].

For the present analysis, the available indicators of nutrient status were selected, such as serum vitamin $B_{12}$, serum folate and serum ferritin. These were analysed using electrochemiluminescence-immunoassay (Elecsys E 2010, Roche Mannheim, Germany). During the survey, the manufacturer changed the method for measuring folate. A conversion factor could not be applied; therefore, separate analyses were performed for the two measurement methods (serum folate 1, serum folate 2).

Furthermore, for this analysis, biochemical and physiological cardiovascular risk factors including blood pressure, total serum cholesterol, low density and high density lipoprotein cholesterol (LDL-C and HDL-C), homocysteine, uric acid, and $\mathrm{HbA1c}$ were also selected. Standardised measurements of systolic and diastolic blood pressure were obtained using an automated oscillometric blood pressure device (Datascope Accutorr Plus) [32, 34, 37]. The means of two independent readings for systolic and diastolic blood pressure were used. Total cholesterol was analysed using an enzymatic assay (cholesterol oxidase-PAP method, Roche Mannheim, Germany). LDL-C and HDL-C were determined directly with a homogenous enzymatic colorimetric assay (Roche Mannheim, Germany). Homocysteine was measured with fluorescent particle immunoassay (Abbot, Wiesbaden, Germany). Uric acid was determined by the uricase-PAP method (Hitachi 917; Roche Mannheim, Germany). HbA1c was analysed using highperformance liquid chromatography (Diastad; Biorad, Munich, Germany) [34].

\section{Assessment of anthropometric markers}

Body height was measured without shoes, with an accuracy of $0.1 \mathrm{~cm}$, by trained staff using a portable Harpenden stadiometer (Holtain Ltd.; Crymych, UK). Body weight was measured while participants were only wearing underwear and no shoes, with an accuracy of $0.1 \mathrm{~kg}$, using a calibrated electronic scale (SECA, Birmingham, UK). Body mass index (BMI) was calculated as body weight (in kilograms) divided by body height squared (in meters) [32].

\section{Assessment of other variables}

Within KiGGS, health related information was assessed through self-administered questionnaires. Regular alcohol consumption was defined as drinking at least one glass of beer, wine, or liquor per week. Smoking habits were assessed with the following question: 'Do you currently smoke?' 'daily', 'several times a week', 'once a week', 'more seldom' or 'no' [38]. This variable was categorized into 'yes' or 'no' (with only those adolescents who never smoke being categorized into "no"). Regarding physical activity, adolescents were asked: 'In your leisure time, how often are you physically active in such a way that you start to sweat or become slightly out of breath?'. The subsequent question: 'How many hours per week?' was used in this analysis.

Medication and supplement use during the last 7 days prior to the interview was determined with a standardised computer-assisted interview conducted by trained physicians. Adolescents were present at the standardised computer-assisted interview where primarily the parents were asked: 'Has your child taken any medications in the last 7 days? Please also mention the use of any ointments, liniments, contraceptive pills, vitamin and mineral supplements, medicinal teas, herbal medicines or homoeopathic medicines'. Parents were asked in advance to bring prescriptions or original containers to the study centre for the purpose of verification $[39,40]$.

\section{Study population}

For the present analysis, all adolescents between 12 and 17 years were selected from the KiGGS survey sample (a total of 2953 boys and 2801 girls). Of these, 292 participants were excluded because they did not provide a blood sample. 263 participants were further excluded because 
they had no, incomplete (more than twenty missing values), or implausibly high total food intake data. That is, if the estimated total food intake exceeded $10 \mathrm{~kg} /$ day, the total beverage intake exceeded $15 \mathrm{l} /$ day, or food intake exceeded $4 \mathrm{~kg} /$ day, combined with beverage intake above 6 1/day. Lastly, two girls who were pregnant were also excluded because pregnancy influences the biomarker profile. Therefore, the final analysis is based on a total sample of 5197 adolescents (2646 boys and 2551 girls).

For specific analyses, persons with missing values (for specific serum variables, for instance) were excluded. Moreover, persons with diabetes mellitus or taking diabetes medication were excluded from the analysis of HbA1c (9 participants); persons taking cardiovascular medication from the analysis of blood pressure (179 participants); persons with vitamin supplement use from the analysis of folate and vitamin $B_{12}(296$ participants); and persons with mineral supplement use from the analysis of ferritin (115 participants). Because hormonal contraceptives influence blood lipids [41], girls with hormonal contraceptive use were excluded from the analysis of blood lipids (397 participants).

\section{Statistical analyses}

Statistical analyses were conducted for boys and girls separately using the SAS version 9.4 (SAS Institute Inc., Cary, USA).

To correct for non-response and disproportional sampling, a weighting factor was used for all analyses. Since the sample is based on a clustered and stratified design, all analyses were performed with complex survey procedures. Differences with $p$-values $<0.05$ were considered statistically significant.

Selected study characteristics were calculated using PROC SURVEYMEANS and PROC SURVEYFREQ. Linear regression models with PROC SURVEYREG were used to examine mean values (with 95\% CI) of biomarkers according to dietary pattern scores. For the analysis, dietary patterns were divided into quintiles and acted as independent variables. The first model (Model 1) was only adjusted for age. The second model (Model 2) was adjusted for age, physical activity (hours per week, continuous), smoking status (yes/no), regular alcohol consumption (yes/no), and mean caloric intake in $\mathrm{kcal} /$ day (continuous). The analyses concerning blood lipids and blood pressure were additionally adjusted for BMI in $\mathrm{kg} / \mathrm{m}^{2}$ in Model 2 . Socioeconomic status influences eating behaviour and, consequently, biomarker status. Therefore, socioeconomic status is part of the chain of causation and was, thus, not considered as a confounder. To examine age differences, additional analyses were conducted stratified by two age groups (12-14 years, 15-17 years) and adjusted according to Model 2. Trend tests were conducted by including the mean score of each pattern quintile as a continuous variable into the models.
Dietary patterns were identified separately for boys and girls using principal component analysis (in SAS: PROC FACTOR method = prin) on 34 food groups. For each food group, the mean amount of grams per day was standardised to a mean of 0 and standard deviation of 1 (z-transformation).

The resulting components were linear combinations of the included variables and explained as much of the variation in the original variables as possible. The components were rotated by an orthogonal transformation (resulting in uncorrelated components) to achieve a simpler structure with greater interpretability. To identify the number of principal components to be retained, the following criteria were used: the criterion of eigenvalues exceeding 1 (the interpretation of this criterion being that each component should explain a larger amount of variance than a single standardised variable in order to be retained), the scree plot (a graphical presentation of eigenvalues), and the interpretability of each component (dietary pattern) [42]. For good interpretability of each component, an adequate number of food groups with high loadings within a component were necessary. According to Hatcher 2007, components with at least 3 'significant' loadings, which were loadings greater than or equal to $|0.4|$, were selected [42]. Each obtained component represents a linear combination of all food groups, which were weighted by their factor loadings. Higher factor loadings indicate that the food variable contributes more to the development of the component. Each participant had a score for all identified dietary patterns, which were standardised to a mean of 0 and a standard deviation of 1 . These scores rank individuals according to the degree to which they conform to each food consumption pattern. The pattern scores were labelled according to the food groups with high loadings.

\section{Results \\ Sample characteristics}

Table 1 illustrates the sample characteristics, stratified by sex. Mean age for the entire sample was 15.1 years. $29.0 \%$ of the boys and $16.5 \%$ of the girls drank at least one glass of beer, wine, or liquor per week. $22.3 \%$ of boys and $22.8 \%$ of girls reported being current smokers. Mean duration of physical activity per week was 8.2 hours among boys and 5.3 hours among girls. $6.6 \%$ of the boys and $4.8 \%$ of the girls used vitamin supplements in the last 7 days, whereas only $3 \%$ of the boys and $1.5 \%$ of the girls used mineral supplements.

\section{Dietary patterns}

Through PCA, three prevailing components (dietary patterns) among boys and two among girls were determined (Table 2). The two components explained 21.5\%, of total variance in food group intake among boys and 15.5\% among girls. 
Table 1 Sample characteristics by sex (mean values or percentages and 95\% Cl)

\begin{tabular}{|c|c|c|c|c|}
\hline & Boys & & Girls & \\
\hline & $N=264$ & & $N=255$ & \\
\hline & Mean & $95 \% \mathrm{Cl}$ & Mean & $95 \% \mathrm{Cl}$ \\
\hline Age (years, mean) & 15.1 & $(15.0-15.1)$ & 15.1 & $(15.0-15.1)$ \\
\hline Regular alcohol consumption (\%) ${ }^{a}$ & 29.0 & $(26.7-31.3)$ & 16.5 & $(14.7-18.4)$ \\
\hline Current smoking (\%) & 22.3 & $(20.4-24.1)$ & 22.8 & $(21.1-24.5)$ \\
\hline Physical activity (hours per week, mean) & 8.2 & $(7.8-8.5)$ & 5.3 & $(5.0-5.5)$ \\
\hline Vitamin supplement use (\%) ${ }^{\mathrm{b}}$ & 6.6 & $(5.6-7.7)$ & 4.8 & $(3.8-5.7)$ \\
\hline Mineral supplement use $(\%)^{\mathrm{b}}$ & 3.0 & $(2.3-3.6)$ & 1.5 & $(1.0-2.0)$ \\
\hline BMI $\left(\mathrm{kg} / \mathrm{m}^{2}\right)$ & 21.2 & $(21.0-21.4)$ & 21.5 & $(21.3-21.7)$ \\
\hline
\end{tabular}

adrinking at least one glass of beer, wine, or liquor per week

${ }^{\mathrm{b}}$ during the last 7 days

Among boys, the first pattern was characterized by higher factor loadings of salty snacks, burger/sausages/ doner kebab, French fries, nuts, desserts/ice cream, pancakes, eggs, and cake/cookies and was, therefore, labelled 'western' dietary pattern. The second pattern among boys was characterized by a typical German diet, with higher factor loadings of white bread, processed meat, meat, margarine, butter, soft drinks, jam, and cheese; hence, it was labelled 'traditional'. The third dietary pattern among boys was labelled 'healthy' because of higher factor loadings of wholemeal bread, fruits, salad vegetables, and other vegetables.

Among girls, the first dietary pattern was characterized by higher factor loadings of salty snacks, burger/sausages/ doner kebab, French fries, dessert/ice cream, pancakes, eggs, cake/cookies similar to the 'western' pattern among boys; it also showed higher loadings of confectionery, potatoes and white bread and was, therefore, labelled 'western and traditional'. The second pattern among girls was associated with higher factor scores of wholemeal bread, fruits, cheese, salad vegetables, and other vegetables and was, thus, labelled 'healthy'.

\section{Dietary patterns and age}

Dietary pattern scores were divided into quintiles, with higher quintiles indicating a higher adherence to this pattern. To characterize the adolescents according to the dietary patterns, mean age per quintile 1,3 , and 5 for every dietary pattern is shown in Table 3 . Boys with higher 'traditional' pattern scores had a higher mean age (Q1: 14.1, Q5: 15.1 years, $p<=0.0001$ ). There were also age differences concerning the other patterns, but these differences were smaller. Boys with higher 'western' pattern scores were older (Q1: 14.6, Q5: 14.9 years $p=0.001)$. In contrast to this finding, girls with higher 'western and traditional' dietary pattern scores were characterized by a lower mean age (Q1: 14.9, Q5: 14.5 years, $p=0.009)$, whereas girls with higher 'healthy' pattern scores had a higher mean age (Q1: 14.5, Q5: 14.8 years, $p=0.005$ ).

\section{Dietary patterns and biomarkers}

Tables 4 and 5 present adjusted means of biomarker levels according to quintiles of dietary patterns scores. Significant $p$-values indicate differences in biomarker levels between dietary pattern quintiles.

Trend tests for the associations between dietary patterns and biomarker profile were conducted in two different models (Table 6). Among boys, in Model 2, the 'western' dietary pattern was negatively associated with ferritin serum concentrations $(p=0.006)$ and diastolic blood pressure $(p=0.002)$. The 'traditional' dietary pattern was negatively associated with serum folate $1(p=0.001)$. The 'healthy' dietary pattern was positively associated with serum folate $1(p=0.001)$ and negatively with serum homocysteine concentrations $(p=0.0003)$.

Among girls, in Model 2, the 'western and traditional' dietary pattern was negatively associated with serum folate $2(p=0.003)$ and positively with homocysteine $(p=$ $0.003)$ concentrations. The 'healthy' dietary pattern was positively associated with folate $(p=0.017)$ and vitamin $B_{12}$ concentrations $(p=0.044)$ and negatively associated with homocysteine $(p=0.0003)$.

In the models adjusted only for age (Model 1 ), there were additional significant associations between dietary patterns and biomarkers: vitamin $\mathrm{B}_{12}$ was negatively associated with the 'western' and positively associated with the 'healthy' pattern among boys $(p=0.049 / 0.017)$. The 'healthy' patterns among both sexes were positively associated with systolic blood pressure $(p=0.045 / 0.015)$ and negatively with HDL-C $(p=0.034 / 0.010)$. In contrast to this, ferritin was not associated with any of the dietary patterns in Model 1.

\section{Dietary patterns and biomarker in different age groups}

Due to the differences in mean ages concerning dietary pattern quintiles, a subgroup analysis for adolescents aged 12 to 14 years and 15 to 17 years was conducted, adjusted according to Model 2 (Additional file 1: Table S1). 
Table 2 Dietary patterns among 12- to 17-year-old adolescents in Germany. Factor loadings for food groups*

\begin{tabular}{|c|c|c|c|c|c|}
\hline & \multicolumn{5}{|c|}{ Dietary patterns } \\
\hline & \multicolumn{3}{|c|}{ Boys $(N=2646)$} & \multicolumn{2}{|l|}{ Girls $(N=2551)$} \\
\hline & 'Western' & 'Traditional' & 'Healthy' & 'Western and traditional' & 'Healthy' \\
\hline Salty snacks & 0.66 & & & 0.57 & \\
\hline Burger/Sausages/Doner kebab & 0.64 & & & 0.54 & \\
\hline French fries & 0.61 & & & 0.57 & \\
\hline Nuts & 0.59 & -0.20 & & & \\
\hline Dessert/Ice-Cream & 0.49 & & & 0.54 & \\
\hline Pancakes & 0.49 & & & 0.47 & \\
\hline Eggs & 0.39 & & & 0.42 & \\
\hline Cake/cookies ${ }^{a}$ & 0.38 & 0.22 & 0.41 & & \\
\hline Soup & 0.34 & & 0.27 & 0.24 & 0.25 \\
\hline Pasta/Rice & 0.33 & & 0.26 & 0.22 & 0.28 \\
\hline Chicken & 0.31 & & & 0.25 & 0.28 \\
\hline Confectionery $^{b}$ & 0.30 & 0.25 & & 0.41 & \\
\hline Other vegetables ${ }^{c}$ & 0.27 & & 0.41 & & 0.39 \\
\hline Potatoes & 0.22 & 0.27 & & 0.39 & \\
\hline Fish & 0.22 & & & 0.27 & \\
\hline Meat & 0.21 & 0.44 & & 0.28 & \\
\hline White bread ${ }^{d}$ & & 0.55 & & 0.36 & \\
\hline Processed meat & & 0.55 & & 0.21 & 0.30 \\
\hline Margarine & & 0.43 & & & 0.32 \\
\hline Butter & & 0.42 & & & 0.22 \\
\hline Soft drinks ${ }^{\mathrm{e}}$ & & 0.39 & -0.25 & 0.32 & \\
\hline $\operatorname{Jam}^{f}$ & & 0.39 & & 0.32 & \\
\hline Cheese $^{g}$ & & 0.36 & 0.34 & & 0.48 \\
\hline Ketchup & & 0.35 & & 0.25 & \\
\hline Milk & & 0.26 & & & 0.25 \\
\hline Breakfast cereals & & 0.26 & & & 0.24 \\
\hline Wholemeal bread & & 0.25 & 0.48 & & 0.52 \\
\hline Fruits $^{\mathrm{h}}$ & & & 0.58 & & 0.49 \\
\hline Salad vegetables & & & 0.54 & & 0.47 \\
\hline Water' & & & 0.33 & & 0.30 \\
\hline Yoghurt other milk products & & & 0.31 & & 0.33 \\
\hline Teaj & & & 0.31 & & 0.31 \\
\hline Juices & & & 0.25 & & 0.29 \\
\hline \multicolumn{6}{|l|}{ Coffee } \\
\hline Variance explained & 11.3 & 5.8 & 4.7 & 9.90 & 5.60 \\
\hline
\end{tabular}

*Factor loadings with absolute values $<0.2$ are not shown for clarity, absolute values $>0.35$ are bold

${ }^{a}$ cake, pastries, cookies

bchocolate, other sweets like candy or fruit gums

ccooked fresh vegetable, canned or frozen vegetable

${ }^{d}$ wheat bread, mixed bread, bread rolls

elemonade, energy drinks

fjam, honey, hazelnut spread

${ }^{9}$ cheese, cream cheese

$h_{\text {fresh and canned fruits }}$

imineral water, tap water

'jerb tea, fruit tea 
Table 3 Age by dietary pattern quintiles (mean and 95\% Cl) among 12- to 17-year-old adolescents in Germany

\begin{tabular}{|c|c|c|c|c|c|}
\hline & Q1 & Q3 & Q5 & $\beta$ & $P$ for trend \\
\hline \multicolumn{6}{|c|}{ Dietary patterns among boys $(N=2646)$} \\
\hline 'Western' & $14.6(14.4-14.7)$ & $14.5(14.3-14.6)$ & $14.9(14.7-15.0)$ & 0.23 & 0.001 \\
\hline 'Traditional' & $14.1(13.9-14.2)$ & $14.6(14.4-14.7)$ & $15.1(15.0-15.3)$ & 0.47 & $<.0001$ \\
\hline 'Healthy' & $14.7(14.6-14.9)$ & $14.6(14.4-14.7)$ & $14.8(14.6-14.9)$ & 0.06 & 0.197 \\
\hline \multicolumn{6}{|c|}{ Dietary patterns among girls $(N=2551)$} \\
\hline 'Western and traditional' & $14.9(14.7-15.0)$ & $14.6(14.5-14.8)$ & $14.5(14.4-14.6)$ & -0.14 & 0.009 \\
\hline 'Healthy' & $14.5(14.4-14.7)$ & $14.5(14.3-14.6)$ & $14.8(14.7-15.0)$ & 0.12 & 0.005 \\
\hline
\end{tabular}

Among boys, significant associations in both age groups were observed between the 'traditional' pattern and folate $1(p=0.009 / 0.014)$ and between the 'healthy' pattern and homocysteine ( $p=0.008 / 0.005)$. The associations between the 'western' and the 'healthy' pattern and vitamin $\mathrm{B}_{12}(p=0.04, p=0.015)$ and between the 'traditional' pattern and homocysteine $(p=0.014)$ were only significant in the younger age group. In contrast to this, the associations between the 'healthy' pattern and folate 1 ( $p=$ $0.007)$ and between the 'western' pattern and ferritin $(p=$ $0.018)$, as well as diastolic blood pressure $(p=0.024)$, were only significant in the older age group.

Among girls, significant associations were observed in both age groups between the 'western and traditional' dietary pattern and homocysteine $(p=0.045, p=0.028)$. In the younger age group, significant associations between the 'western and traditional' $(p=0.007)$ and the 'healthy' dietary pattern $(p=0.015)$ and folate 2 and between the 'healthy' dietary pattern and vitamin $\mathrm{B}_{12}(p=0.008)$ were observed. Associations between the 'healthy' dietary pattern and ferritin $(p=0.027)$ and homocysteine $(p=0.006)$, however, were only seen in the older age group.

\section{Discussion}

In a representative population of German adolescents, we identified three major dietary patterns among boys and two among girls. Adolescents with higher scores in the 'healthy' dietary patterns had a better nutrient profile. Concerning cardiovascular risk factors, only few significant associations were found in this young population. The most pronounced was the association with homocysteine.

We observed age group differences (12 to 14 years vs 15 to 17 years) in the associations between dietary patterns and biomarkers. It also appeared that the less healthy patterns were more common in older boys. In contrast to this, girls with greater adherence to more unfavourable patterns were younger and those with greater adherence to the healthy pattern were older. Since this was a cross-sectional study, these findings should be further investigated in longitudinal analyses.
The higher importance of a less healthy dietary pattern among older adolescents, similar to the German boys, was also observed in Greece [43]. Healthier dietary patterns among younger adolescents were observed in Australia [30] and Greece [43]. In contrast to this, in Brazil a 'western' pattern was more common among adolescents below 15 years of age [44].

The role of homocysteine as being an independent risk factor for the pathogenesis of atherosclerosis is controversial in the literature [45]. It is well-established that vitamin $B_{12}$ and folate are required for decomposition of homocysteine. In former studies, an inverse association between homocysteine levels and folate, as well as vitamin $B_{12}$, was observed [46]. Results of our study were in accordance with this biochemical relationship. Those adolescents with higher folate and vitamin $B_{12}$ concentrations had lower homocysteine concentrations (Model 2, Table 6). Since vegetables, fruits, and wholemeal bread are important sources of folate, the higher serum concentrations among adolescents with higher 'healthy' pattern scores were expected. Boys with higher 'western' pattern scores were characterised by lower vitamin $\mathrm{B}_{12}$ serum levels and girls with higher 'healthy' pattern scores by higher serum levels (Table 4). This is in accordance with the higher intake of milk products (cheese, milk, yoghurt and other milk products) and also of margarine (which is to some extend enriched with vitamin $B_{12}$ in Germany) among girls with higher 'healthy' pattern scores. These food groups were less important for boys with higher 'western' pattern scores (factor loadings < 0.2 , Table 2).

Among 15 to 17 years old boys, the 'western' pattern was associated with lower ferritin levels and among 15 to 17 years old girls, the 'healthy' pattern was associated with higher ferritin levels. In a previous subgroup analysis, we had determined the major food sources for ferritin intake [47]. These were bread, sweets, juices, and meat/bowels among boys and bread, juices, vegetables, and sweets among girls. This is in accordance with the factor loadings in these food groups (except for confectionary), which were lower in the 'western' pattern (bread and juices $<0.2$, meat 0.21 , and confectionery 0.3 ) than in the 'healthy' 


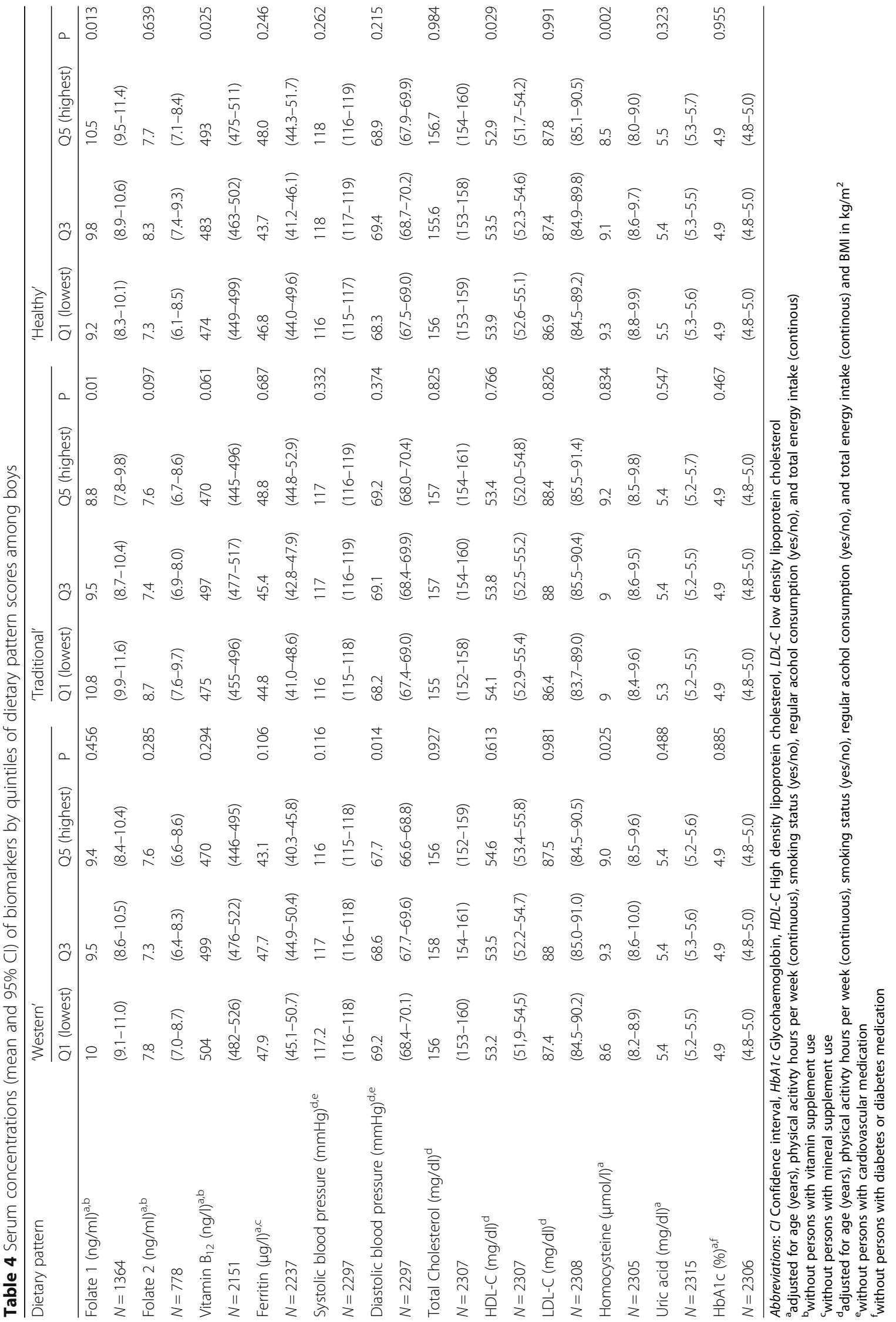


Table 5 Serum concentrations (mean and 95\% Cl) of biomarkers by quintiles of dietary pattern scores among girls

\begin{tabular}{|c|c|c|c|c|c|c|c|c|}
\hline \multirow[t]{2}{*}{ Dietary pattern } & \multicolumn{4}{|c|}{ 'Western and traditional' } & \multicolumn{4}{|l|}{ 'Healthy' } \\
\hline & Q1 (lowest) & Q3 & Q5 (highest) & $P$ & Q1 (lowest) & Q3 & Q5 (highest) & $\mathrm{P}$ \\
\hline Folate $1(\mathrm{ng} / \mathrm{ml})^{\mathrm{a}, \mathrm{b}}$ & 10 & 10.2 & 9.4 & 0.055 & 9.9 & 9.4 & 9.4 & 0.452 \\
\hline$N=1203$ & $(9.3-10.6)$ & $(9.5-11.0)$ & $(8.6-10.2)$ & & $(9.1-10.7)$ & $(8.8-10.0)$ & $(8.7-10.1)$ & \\
\hline Folate $2(\mathrm{ng} / \mathrm{ml})^{\mathrm{a}, \mathrm{b}}$ & 8.0 & 7.2 & 5.8 & 0.017 & 6.8 & 7.4 & 8.6 & 0.039 \\
\hline$N=714$ & $(7.2-8.7)$ & $(6.7-7.7)$ & $(4.8-6.8)$ & & $(6.1-7.4)$ & $(6.8-7.9)$ & $(7.0-10.2)$ & \\
\hline Vitamin $B_{12}(n g / l)^{a, b}$ & 520 & 498 & 486 & 0.474 & 490 & 500 & 528 & 0.385 \\
\hline$N=1920$ & $(496-544)$ & $(477-519)$ & $(453-518)$ & & $(468-512)$ & $(475-524)$ & $(498-558)$ & \\
\hline Ferritin $\left(\mu \mathrm{g} / \mathrm{l}^{\mathrm{a}, \mathrm{c}}\right.$ & 34.5 & 31.6 & 32.6 & 0.252 & 31.5 & 34.1 & 35.6 & 0.092 \\
\hline$N=1992$ & $(31.9-37.2)$ & $(29.6-33.7)$ & $(29.8-35.5)$ & & $(29.2-33.8)$ & $(31.2-36.9)$ & $(32.2-38.9)$ & \\
\hline Systolic blood pressure $(\mathrm{mmHg})^{\mathrm{d}, \mathrm{e}}$ & 113 & 114 & 112 & 0.125 & 112 & 114 & 113 & 0.255 \\
\hline$N=2024$ & $(112-114)$ & $(113-115)$ & $(111-113)$ & & $(111-113)$ & $(112-115)$ & $(112-115)$ & \\
\hline Diastolic blood pressure $(\mathrm{mmHg})^{\mathrm{d}, \mathrm{e}}$ & 68 & 68.3 & 67.7 & 0.757 & 67.5 & 67.9 & 68.3 & 0.669 \\
\hline$N=2024$ & $(67.1-68.9)$ & $(67.5-69.1)$ & $(66.7-68.6)$ & & $(66.7-68.3)$ & $(67.2-68.6)$ & $(67.3-69.3)$ & \\
\hline Total Cholesterol $(\mathrm{mg} / \mathrm{dl})^{\mathrm{d}, \mathrm{f}}$ & 164 & 161 & 164 & 0.443 & 165 & 160 & 162 & 0.173 \\
\hline$N=1743$ & $(161-168)$ & $(157-164)$ & $(160-169)$ & & $(162-169)$ & $(157-163)$ & $(159-166)$ & \\
\hline $\mathrm{HDL}-\mathrm{C}(\mathrm{mg} / \mathrm{dll})^{\mathrm{d}, \mathrm{f}}$ & 58.1 & 56.9 & 59 & 0.355 & 58.7 & 58.4 & 58.2 & 0.665 \\
\hline$N=1743$ & $(56.8-59.3)$ & $(55.6-58.2)$ & $(57.1-61.0)$ & & $(57.1-60.2)$ & $(57.0-59.7)$ & $(56.7-59.6)$ & \\
\hline $\operatorname{LDL}-C(\mathrm{mg} / \mathrm{dl})^{\mathrm{d}, \mathrm{f}}$ & 93.3 & 91.6 & 92 & 0.891 & 94 & 89 & 92.7 & 0.112 \\
\hline$N=1743$ & $(90.2-98.4)$ & $(88.4-94.8)$ & $(88.1-96.0)$ & & $(91.0-97.0)$ & $(86.3-91.6)$ & $(89.0-96.3)$ & \\
\hline Homocysteine $(\mu \mathrm{mol} / \mathrm{l})^{\mathrm{a}}$ & 7.6 & 7.6 & 8.1 & 0.029 & 8.0 & 7.5 & 7.4 & 0.002 \\
\hline$N=2021$ & $(7.3-7.9)$ & $(7.3-7.9)$ & $(7.7-8.5)$ & & $(7.7-8.3)$ & $(7.2-7.8)$ & $(7.0-7.8)$ & \\
\hline Uric acid $(\mathrm{mg} / \mathrm{dl})^{\mathrm{a}}$ & 4.4 & 4.3 & 4.3 & 0.586 & 4.3 & 4.3 & 4.4 & 0.204 \\
\hline$N=2036$ & $(4.2-4.5)$ & $(4.1-4.4)$ & $(4.1-4.5)$ & & $(4.1-4.4)$ & $(4.1-4.4)$ & $(4.2-4.6)$ & \\
\hline $\mathrm{HbA} 1 \mathrm{c}(\%)^{\mathrm{a}, \mathrm{g}}$ & 4.8 & 4.9 & 4.9 & 0.391 & 4.8 & 4.8 & 4.8 & 0.687 \\
\hline$N=2023$ & $(4.8-4.9)$ & $(4.8-4.9)$ & $(4.8-5.0)$ & & $(4.8-4.9)$ & $(4.8-4.9)$ & $(4.7-4.9)$ & \\
\hline
\end{tabular}

Abbreviations: Cl Confidence interval, HbA1c Glycohaemoglobin, HDL-C High density lipoprotein cholesterol, LDL-C low density lipoprotein cholesterol a adjusted for age (years), hours physical acitivty per week (continuous), smoking status (yes/no), regular alcohol consumption (yes/no), and total energy intake (continous)

bithout persons with vitamin supplement use

cwithout persons with mineral supplement use

dadjusted for age (years), hours physical acitivty per week (continuous), smoking status (yes/no), regular alcohol consumption (yes/no), and total energy intake (continous) and BMI

e without persons with cardiovascular medication

fwithout persons with hormonal contraceptive use

${ }^{g}$ without persons with diabetes or diabetes medication

pattern among girls (wholemeal bread 0.52, other vegetables 0.39 , juices 0.29 , and confectionery $<0.2$ ). In a sensitivity analysis, we further adjusted for BMI, this did not change the results substantially (data not shown).

A negative relationship between the 'western' dietary pattern and diastolic blood pressure was observed in both models with different adjustments. In age-stratified analyses, the association was only significant for 15 to 17 year old boys. Corresponding to the food groups with higher (red meat, confectionery, dessert/ice cream) and lower factor loading (fruits, vegetables, whole grain, and fish), the direction of this finding was not expected. On the other hand, this pattern was also characterized by nuts and chicken, food groups that were recommended in the Dietary Approach to Stop Hypertension (DASH) [48]. However, the absolute differences between dietary pattern quintiles were rather small $(1.5 \mathrm{mmHg}$ between the 1 th and the $5^{\text {th }}$ quintile, Table 4). Furthermore, blood pressure is not influenced by nutrition only; therefore, some major confounders were accounted for by adjustment (BMI, physical activity, alcohol consumption, age) and by gender stratified analysis. However, measurement of physical activity is a difficult concept and represents energy expenditure due to body movement only partially. Therefore, residual confounding could still be present.

In a previous analysis, we considered the association between healthy diet indices based on German Food 


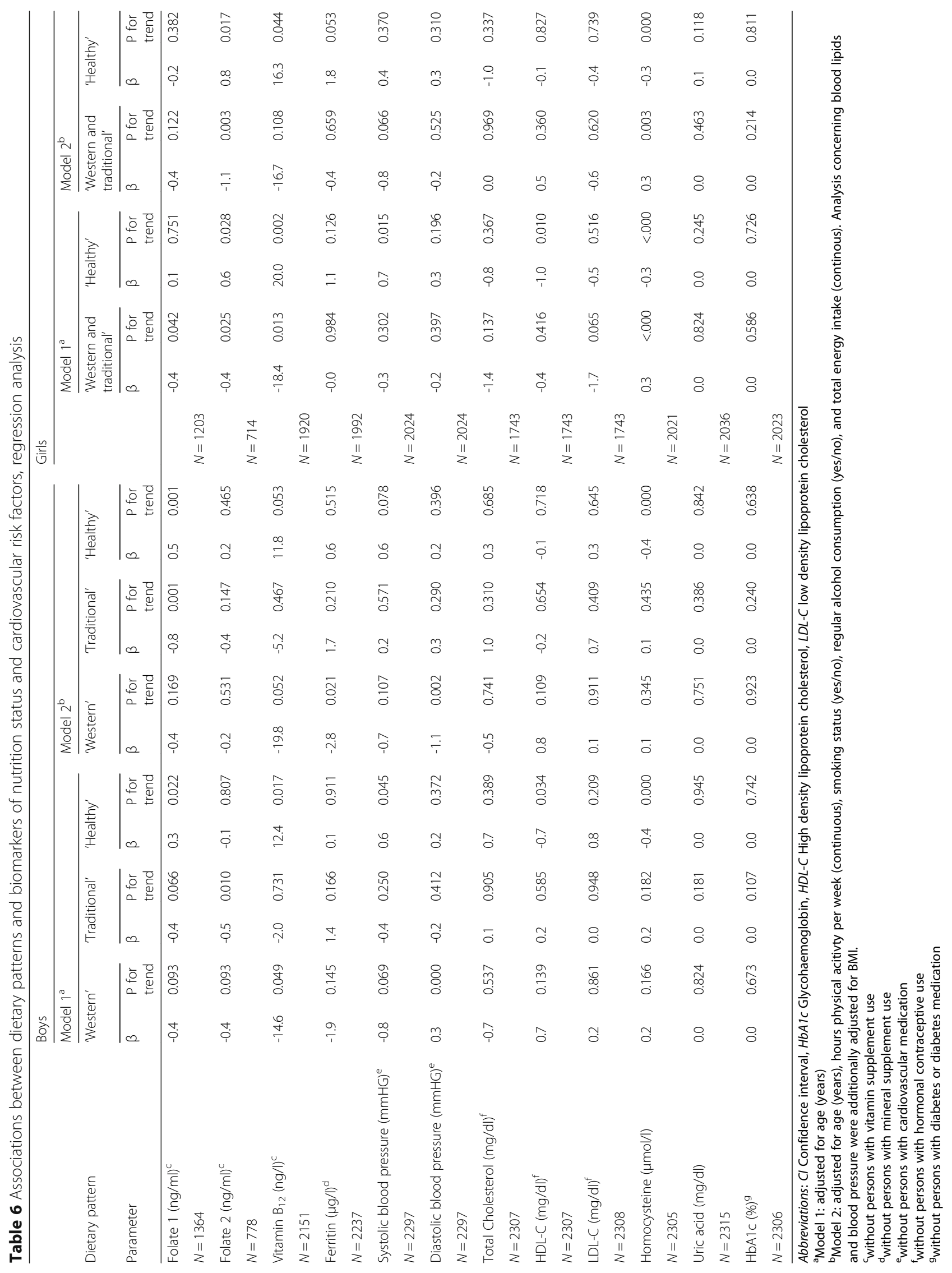


Intake Recommendations and biomarkers within this same population [49]. We found that some of the indices were associated with biomarker profiles. The advantage of this current analysis of dietary patterns determined by a data driven approach is that these patterns more objectively represent prevailing eating habits of the population.

Among adults, many previous studies have shown that dietary patterns are related to biomarkers of cardiovascular risk [23-26, 50]. In contrast to this, analyses of dietary patterns and biomarkers among adolescents are rare. Associations between dietary patterns and blood pressure have been analyzed in two representative surveys among adolescents elsewhere. In Australia, a dietary pattern that was characterized by fruits, salad, cereals, and fish was negatively associated with diastolic blood pressure among adolescents aged 16 to 18 years [30]. In Tunisia, the 'meat-fish' pattern was positively associated with diastolic blood pressure among boys [31]. In Finland, a positive association between systolic blood pressure and the 'traditional' pattern was found in a cross sectional analysis of cohort data [28]. Other cohort studies found no association between dietary patterns and systolic $[27,51]$ or diastolic blood pressure [27]. Thus, it seems that associations between dietary patterns and blood pressure were seldom observed in this young age group and were found only in subgroups, which is in accordance with our study.

In former studies, patterns like 'fruit and vegetable' [27] and 'healthy' among boys [29] were associated with more favorable blood lipids concentrations. Furthermore, the 'traditional' pattern in Finland [28] and the 'sugar foods' and 'fats and pasta' pattern in the U.S. [27] were associated with less beneficial blood lipids concentrations. These analyses were based on cohort studies. To our knowledge, this is the first analysis of dietary pattern conducted with PCA or factor analysis and blood lipids in this age group using a nationwide representative survey. Among adolescents in Germany, we did not observe associations between dietary patterns and serum lipids. In contrast to previous studies [27-29], we excluded girls with hormonal contraceptive use in the analysis of blood lipids because they have higher blood lipids concentrations caused by the medication [41]. Hormonal contraceptive use is not a typical confounder because the use is not associated with the dietary patterns. Overall, $16 \%$ of the girls in this age group used this medication, with the highest percentage among 16 to 17 years old girls (30\%).

The negative association between healthy dietary patterns and homocysteine concentrations was also found in a cohort study among 9 to 24 years old female Finns [28] and among young adults in Northern Ireland [52]. The 'traditional' dietary pattern was not associated with homocysteine concentrations in Finland. Whereas, in Northern Ireland, the 'western' pattern was positive associated with homocysteine [52], similar to the 'western and traditional' pattern in Germany.

Biomarkers are objectively measured and can be evaluated as indicators of nutrient supply. To our knowledge, there are no other studies in this age group that have analyzed the associations between dietary patterns determined through PCA or factor analysis and vitamin and mineral serum concentrations. In a cohort study among young adults in Northern Ireland, higher red cell folate and vitamin $B_{12}$ serum concentrations were associated with the 'healthy' dietary pattern among men and with the 'traditional' dietary pattern among women [53]. Thus, the 'traditional' dietary pattern in Northern Ireland was characterized by a more favorable nutrient status, whereas the 'traditional' dietary pattern in Germany was not. Associations concerning the 'healthy' pattern were in the same direction as in our study.

Strengths of this study include data obtained from a large, nationally-representative, population based sample. Furthermore, KiGGS provides a broad spectrum of data on biochemical parameters and anthropometric measures, all assessed by trained staff, as well as further information on participants' behaviors, such as on physical activity levels and medication use. In addition, we used a validated FFQ to examine food intake. The percentage of variance explained by the dietary patterns was within the range of what has been previously reported in other studies that studied dietary patterns of adolescents [30, 43, 54, 55].

However, there are several limitations which have to be considered. With an FFQ, only a predefined selection of foods and food groups can be assessed. Therefore, the consumption of other foods is unknown. There may also be a certain overlap of food groups if they are defined or perceived too broadly. In addition, portion sizes and, thus, energy intake can only be estimated roughly. These limitations occur in all food intake data assessed with an FFQ. However, in a sub-sample of the KiGGS study population, a more comprehensive modified dietary history interview (DISHES) was conducted several months after the KiGGS survey. In this subgroup, we identified very similar dietary patterns [56]. In comparison to this study, there were only some differences in food groups with higher factor loadings belonging to one pattern. These differences can be explained by differences in the dietary assessment methods, e.g. in the FFQ, pizza, vegetable oil, mushrooms, and alcoholic drinks were not assessed while rice and pasta were asked as one food item; however, with the DISHES data, these foods were analyzed separately. Overall, the FFQ seems to be appropriate to determine dietary patterns in this population. However, inclusion of some more food groups would be useful.

Limitations of using the PCA are the prior classification of food items into food groups, the decision of the number of factors extracted, and the labeling of factors, which can 
be subjective decisions. To enhance comparability with other studies, similar methodological steps were used in the extraction of the dietary patterns as those utilized in other studies [43, 57].

Further research is still necessary to evaluate tracking of dietary patterns during the life course. Although the present analysis was cross-sectional, future longitudinal analyses are planned and data collection for the follow-up is ongoing.

\section{Conclusions}

In conclusion, our cross-sectional analysis identified that some associations between dietary patterns and biomarkers of nutrient status and cardiovascular risk already become evident among adolescents. Therefore, dietary patterns can influence health status. Dietary patterns adopted during adolescence may track into adulthood, and can, therefore, be important for health outcomes later in life. Since eating habits are a modifiable risk factor for cardiovascular diseases, public health policies and health promotion programs should target adolescents to establish healthy dietary practices for life.

\section{Additional file}

Additional file 1: Table S1. Associations between dietary patterns and biomarkers of nutrition status and cardiovascular risk factors, regression analysis. (DOCX $27 \mathrm{~kb}$ )

\section{Abbreviations}

BMI: Body mass index; Cl: Confidence interval; CVD: Cardiovascular diseases; DISHES: Dietary interview software for health examination studies; FFQ: Food frequency questionnaire; HbA1c: Glycohaemoglobin; HDL-C: High density lipoprotein cholesterol; KiGGS: Health Interview and Examination Survey for Children and Adolescents in Germany; LDL-C: Low density lipoprotein cholesterol; PCA: Principal component analysis

\section{Acknowledgments}

We thank the adolescents who participated in this study, and their parents for filling in the questionnaires and answering our questions.

\section{Funding}

KiGGS was funded by the German Federal Ministry of Health, the German Federal Ministry of Education and Research (grant number 01EH0201) and the Robert Koch Institute.

\section{Availability of data and material}

KiGGS data are available as public use file at RKI homepage (http://www.rki.de/ DE/Content/Gesundheitsmonitoring/Forschungsdatenzentrum/

forschungsdatenzentrum_node.html).

\section{Authors' contributions}

$A R, S T, J R$ and GBMM designed the analysis plan. AR analysed the data, drafted the manuscript, and wrote the final version. MR, JT and GBMM contributed to the construction of variables. GBMM was involved in the design and conduction of KiGGS and responsible for the design of the FFQ. All authors contributed to writing and revising the manuscript and read and approved the final manuscript.

\section{Competing interests}

The authors declare that they have no competing interests.
Consent for publication

Not applicable.

\section{Ethics approval and consent to participate}

KiGGS was approved by the Federal Office for Data Protection and the Charité-Universitätsmedizin Berlin ethics committee. Participants aged 14 years or older and all parents provided written informed consent before the interview and examination procedures.

\section{Author details}

'Department of Epidemiology and Health Monitoring, Robert Koch Institute Berlin, Post box 6502 61D-13302 Berlin, Germany. ${ }^{2}$ Chair of Marketing and Consumer Research, TUM School of Management München, Technische Universität, Alte Akademie 16, D-85350 Freising, Germany. ${ }^{3}$ Department of Food Economics and Consumption Studies, Christian-Albrechts-Universität Kiel, Olshausenstraße 40, D-24098 Kiel, Germany.

Received: 4 August 2016 Accepted: 19 December 2016

Published online: 05 January 2017

\section{References}

1. Spear BA. Adolescent growth and development. J Am Diet Assoc. 2002; 102(3 Suppl):S23-9.

2. Banfield EC, Liu Y, Davis JS, Chang S, Frazier-Wood AC. Poor Adherence to US Dietary Guidelines for Children and Adolescents in the National Health and Nutrition Examination Survey Population. J Acad Nutr Diet. 2016;116(1): 21-7. doi:10.1016/j.jand.2015.08.010.

3. Doidge JC, Segal L. Most Australians do not meet recommendations for dairy consumption: findings of a new technique to analyse nutrition surveys. Aust N Z J Public Health. 2012;36(3):236-40. doi:10.1111/j.1753-6405.2012.00870.x.

4. Munoz KA, Krebs-Smith SM, Ballard-Barbash R, Cleveland LE. Food intakes of US children and adolescents compared with recommendations. Pediatrics. 1997:100(3 Pt 1):323-9.

5. Neumark-Sztainer D, Story M, Resnick MD, Blum RW. Lessons learned about adolescent nutrition from the Minnesota Adolescent Health Survey. J Am Diet Assoc. 1998;98(12):1449-56. doi:10.1016/S0002-8223(98)00329-0.

6. Serra-Majem L, Ribas-Barba L, Salvador G, Serra J, Castell C, Cabezas C, et al. Compliance with dietary guidelines in the Catalan population: basis for a nutrition policy at the regional level (the PAAS strategy). Public Health Nutr. 2007;10(11A):1406-14. doi:10.1017/S1368980007001012.

7. Troiano RP, Briefel RR, Carroll MD, Bialostosky K. Energy and fat intakes of children and adolescents in the united states: data from the national health and nutrition examination surveys. Am J Clin Nutr. 2000;72(5 Suppl):1343S-53S.

8. Zapata LB, Bryant CA, McDermott RJ, Hefelfinger JA. Dietary and physical activity behaviors of middle school youth: the youth physical activity and nutrition survey. J Sch Health. 2008:78(1):9-18. doi:10.1111/j.1746-1561.2007.00260x. quiz 65-7.

9. Mensink GB, Kleiser C, Richter A. Food consumption of children and adolescents in Germany. Results of the German Health Interview and Examination Survey for Children and Adolescents (KiGGS). Bundesgesundheitsblatt Gesundheitsforschung Gesundheitsschutz. 2007; 50(5-6):609-23. doi:10.1007/s00103-007-0222-x.

10. Richter A, Vohmann C, Stahl A, Heseker H, Mensink GBM. Der aktuelle Lebensmittelverzehr von Kindern und Jugendlichen in Deutschland. Ernährungsumschau. 2008;55(1/08):28-36.

11. Kaikkonen JE, Mikkila V, Magnussen CG, Juonala M, Viikari JS, Raitakari OT. Does childhood nutrition influence adult cardiovascular disease risk?-insights from the Young Finns Study. Ann Med. 2013;45(2):120-8. doi:10.3109/07853890.2012.671537.

12. Lauer RM, Lee J, Clarke WR. Factors affecting the relationship between childhood and adult cholesterol levels: the Muscatine Study. Pediatrics. 1988;82(3):309-18.

13. Tracy RE, Newman 3rd WP, Wattigney WA, Berenson GS. Risk factors and atherosclerosis in youth autopsy findings of the Bogalusa Heart Study. Am J Med Sci. 1995:310 Suppl 1:S37-41.

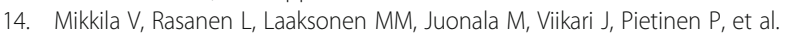
Long-term dietary patterns and carotid artery intima media thickness: the Cardiovascular Risk in Young Finns Study. Br J Nutr. 2009;102(10):1507-12. doi:10.1017/S000711450999064X

15. Ambrosini GL, Emmett PM, Northstone K, Jebb SA. Tracking a dietary pattern associated with increased adiposity in childhood and adolescence. Obesity (Silver Spring). 2014;22(2):458-65. doi:10.1002/oby.20542. 
16. Tuttle C. Childhood and Adolescence. Essentials of human nutrition. Oxford, New York, Tokyo: Oxford University Press; 1999.

17. Gassin AL. Helping to promote healthy diets and lifestyles: the role of the food industry. Public Health Nutr. 2001;4(6A):1445-50.

18. Leach H. Food Habits. Essentials of human nutrition. Oxford, New York, Tokyo: Oxford University Press; 1999.

19. Birch LL, Fisher JO. Development of eating behaviors among children and adolescents. Pediatrics. 1998;101 (3 Pt 2):539-49.

20. Nicklaus S. Development of food variety in children. Appetite. 2009;52(1): 253-5. doi:10.1016/j.appet.2008.09.018.

21. Nicklaus S, Boggio V, Chabanet C, Issanchou S. A prospective study of food variety seeking in childhood, adolescence and early adult life. Appetite. 2005;44(3):289-97. doi:10.1016/j.appet.2005.01.006.

22. Truthmann J, Mensink GB, Richter A. Relative validation of the KiGGS Food Frequency Questionnaire among adolescents in Germany. Nutr J. 2011;10(1): 133. doi:10.1186/1475-2891-10-133.

23. Eilat-Adar S, Mete M, Nobmann ED, Xu J, Fabsitz RR, Ebbesson SO, et al. Dietary patterns are linked to cardiovascular risk factors but not to inflammatory markers in Alaska Eskimos. J Nutr. 2009;139(12):2322-8. doi:10.3945/jn.109.110387.

24. Esmaillzadeh A, Kimiagar M, Mehrabi Y, Azadbakht L, Hu FB, Willett WC. Dietary patterns, insulin resistance, and prevalence of the metabolic syndrome in women. Am J Clin Nutr. 2007;85(3):910-8.

25. Panagiotakos DB, Pitsavos C, Skoumas Y, Stefanadis C. The association between food patterns and the metabolic syndrome using principal components analysis: The ATTICA Study. J Am Diet Assoc. 2007;107(6): 979-87. doi:10.1016/j.jada.2007.03.006. quiz 97.

26. Heidemann C, Scheidt-Nave C, Richter A, Mensink GB. Dietary patterns are associated with cardiometabolic risk factors in a representative study population of German adults. Br J Nutr. 2011;106(8):1253-62. doi:10.1017/S0007114511001504.

27. Nicklas TA, Webber LS, Thompson B, Berenson GS. A multivariate model for assessing eating patterns and their relationship to cardiovascular risk factors: the Bogalusa Heart Study. Am J Clin Nutr. 1989;49(6):1320-7.

28. Mikkilä $\vee$, Räsänen $L$, Raitakari OT, Marniemi J, Pietinen P, Rönnemaa T, et al. Major dietary patterns and cardiovascular risk factors from childhood to adulthood. The Cardiovascular Risk in Young Finns Study. Br J Nutr. 2007; 98(1):218-25.

29. Ambrosini GL, Huang RC, Mori TA, Hands BP, O'Sullivan TA, de Klerk NH, et al. Dietary patterns and markers for the metabolic syndrome in Australian adolescents. Nutr Metab Cardiovasc Dis. 2010;20(4):274-83. doi:10.1016/j. numecd.2009.03.024.

30. McNaughton SA, Ball K, Mishra GD, Crawford DA. Dietary patterns of adolescents and risk of obesity and hypertension. J Nutr. 2008;138(2):364-70.

31. Aounallah-Skhiri H, Traissac P, El Ati J, Eymard-Duvernay S, Landais E, Achour $\mathrm{N}$, et al. Nutrition transition among adolescents of a south-Mediterranean country: dietary patterns, association with socio-economic factors, overweight and blood pressure. A cross-sectional study in Tunisia. Nutr J. 2011;10:38. doi:10.1186/1475-2891-10-38.

32. Kurth BM, Kamtsiuris $P$, Holling $H$, Schlaud M, Dolle R, Ellert U, et al. The challenge of comprehensively mapping children's health in a nation-wide health survey: design of the German KiGGS-Study. BMC Public Health. 2008; 8:196. doi:10.1186/1471-2458-8-196.

33. Holling H, Kamtsiuris $P$, Lange M, Thierfelder W, Thamm M, Schlack R. The German Health Interview and Examination Survey for Children and Adolescents (KiGGS): study management and conduct of fieldwork. Bundesgesundheitsblatt Gesundheitsforschung Gesundheitsschutz. 2007; 50(5-6):557-66. doi:10.1007/s00103-007-0216-8.

34. Thierfelder W, Dortschy R, Hintzpeter B, Kahl H, Scheidt-Nave C. Biochemical measures in the German Health Interview and Examination Survey for Children and Adolescents (KiGGS). Bundesgesundheitsblatt Gesundheitsforschung Gesundheitsschutz. 2007;50(5-6):757-70. doi:10.1007/s00103-007-0238-2.

35. Mensink GB, Burger M. What do you eat? Food frequency questionnaire for children and adolescents. Bundesgesundheitsblatt Gesundheitsforschung Gesundheitsschutz. 2004;47(3):219-26. doi:10.1007/s00103-003-0794-z.

36. Mensink GB, Bauch A, Vohmann C, Stahl A, Six J, Kohler S, et al. EskiMo - the nutrition module in the German Health Interview and Examination Survey for Children and Adolescents (KiGGS). Bundesgesundheitsblatt Gesundheitsforschung Gesundheitsschutz. 2007;50(5-6):902-8. doi:10.1007/s00103-007-0254-2.

37. Neuhauser HK, Rosario AS, Thamm M, Ellert U. Prevalence of children with blood pressure measurements exceeding adult cutoffs for optimal blood pressure in Germany. Eur J Cardiovasc Prev Rehabil. 2009;16(2):195-200.
38. Lampert T, Thamm M. Consumption of tobacco, alcohol and drugs among adolescents in Germany. Results of the German Health Interview and Examination Survey for Children and Adolescents (KiGGS). Bundesgesundheitsblatt Gesundheitsforschung Gesundheitsschutz. 2007; 50(5-6):600-8. doi:10.1007/s00103-007-0221-y.

39. Du Y, Knopf H. Self-medication among children and adolescents in Germany: results of the National Health Survey for Children and Adolescents (KiGGS). Br J Clin Pharmacol. 2009;68(4):599-608. doi:10.1111/j.1365-2125.2009.03477x.x.

40. Knopf H. Medicine use in children and adolescents. Data collection and first results of the German Health Interview and Examination Survey for Children and Adolescents (KiGGS). Bundesgesundheitsblatt Gesundheitsforschung Gesundheitsschutz. 2007;50(5-6):863-70. doi:10.1007/s00103-007-0249-z.

41. Naz F, Jyoti S, Akhtar N, Afzal M, Siddique YH. Lipid profile of women using oral contraceptive pills. Pak J Biol Sci. 2012;15(19):947-50.

42. Hatcher L. A Step-by-Step Approach to Using the SAS System for Factor Analysis and Structural Equation Modeling. 9. ed. SAS Institute Inc., SAS Campus Drive, Cary, North Carolina; 2007.

43. Kourlaba G, Panagiotakos DB, Mihas K, Alevizos A, Marayiannis K, Mariolis A, et al. Dietary patterns in relation to socio-economic and lifestyle characteristics among Greek adolescents: a multivariate analysis. Public Health Nutr. 2009;12(9):1366-72. doi:10.1017/S1368980008004060.

44. Rodrigues PR, Pereira RA, Cunha DB, Sichieri R, Ferreira MG, Vilela AA, et al. Factors associated with dietary patterns in adolescents: a school-based study in Cuiaba, Mato Grosso. Rev Bras Epidemiol. 2012;15(3):662-74.

45. Pang X, Liu J, Zhao J, Mao J, Zhang X, Feng L, et al. Homocysteine induces the expression of C-reactive protein via NMDAr-ROS-MAPK-NF-kappaB signal pathway in rat vascular smooth muscle cells. Atherosclerosis. 2014;236(1): 73-81. doi:10.1016/j.atherosclerosis.2014.06.021.

46. McKinley MC. Nutritional aspects and possible pathological mechanisms of hyperhomocysteinaemia: an independent risk factor for vascular disease. Proc Nutr Soc. 2000;59(2):221-37.

47. Mensink GBM, Heseker H, Richter A, Stahl A, Vohmann C. Ernährungsstudie als KiGGS-Modul (EsKiMo). Forschungsbericht. Berlin, Paderborn: Robert Koch-Institut, Universtität Paderborn; 2007.

48. U. S. Department of Health and Human Services, National Heart, Lung, and Blood Institute,. Your Guide to Lowering Your Blood Pressure with DASH. NIH Publication. 2006;no. 06-4082.

49. Truthmann J, Richter A, Thiele S, Drescher L, Roosen J, Mensink GB. Associations of dietary indices with biomarkers of dietary exposure and cardiovascular status among adolescents in Germany. Nutr Metab (Lond). 2012;9(1):92. doi:10.1186/1743-7075-9-92.

50. Niu K, Momma H, Kobayashi Y, Guan L, Chujo M, Otomo A, et al. The traditional Japanese dietary pattern and longitudinal changes in cardiovascular disease risk factors in apparently healthy Japanese adults. Eur J Nutr. 2015. doi:10.1007/s00394-015-0844-y.

51. Ambrosini GL, Oddy WH, Robinson M, O'Sullivan TA, Hands BP, de Klerk NH, et al. Adolescent dietary patterns are associated with lifestyle and family psycho-social factors. Public Health Nutr. 2009:12(10):1807-15. doi:10.1017/S1368980008004618.

52. McCourt HJ, Draffin CR, Woodside JV, Cardwell CR, Young IS, Hunter SJ, et al. Dietary patterns and cardiovascular risk factors in adolescents and young adults: the Northern Ireland Young Hearts Project. Br J Nutr. 2014;112(10): 1685-98. doi:10.1017/S0007114514002682.

53. McCourt HJ, Whittle CR, Young IS, Hunter SJ, Murray LJ, Boreham AM, et al. Dietary patterns and cardiovascular risk factors in young adults: the Young Hearts Project, Northern Ireland. Proc Nutr Soc. 2010;69:E365.

54. Romero-Polvo A, Denova-Gutierrez E, Rivera-Paredez B, Castanon S, Gallegos-Carrillo K, Halley-Castillo E, et al. Association between dietary patterns and insulin resistance in Mexican children and adolescents. Ann Nutr Metab. 2012:61(2):142-50, doi:10:1159/000341493.

55. Naja F, Hwalla N, Itani L, Karam S, Mehio Sibai A, Nasreddine L. A Western dietary pattern is associated with overweight and obesity in a national sample of Lebanese adolescents (13-19 years): a cross-sectional study. Br J Nutr. 2015:1-11. doi:10.1017/S0007114515003657.

56. Richter A, Heidemann C, Schulze MB, Roosen J, Thiele S, Mensink GB. Dietary patterns of adolescents in Germany-associations with nutrient intake and other health related lifestyle characteristics. BMC Pediatr. 2012;12:35. do: 10.1186/1471-2431-12-35

57. Cutler GJ, Flood A, Hannan P, Neumark-Sztainer D. Major patterns of dietary intake in adolescents and their stability over time. J Nutr. 2009;139(2):323-8. doi:10.3945/jn.108.090928. 